\title{
Multidisciplinary Approach to Decrease In-Hospital Delay for Stroke Thrombolysis
}

\author{
Sang-Beom Jeon, ${ }^{a}$ Seung Mok Ryoo, ${ }^{\mathrm{b}}$ Deok Hee Lee, ${ }^{\mathrm{c}}$ Sun U. Kwon, ${ }^{\mathrm{a}}$ Seongsoo Jang, ${ }^{\mathrm{d}}$ Eun-Jae Lee, ${ }^{\mathrm{a}}$ \\ Sang Hun Lee, ${ }^{\mathrm{b}}$ Jung Hee Han, ${ }^{\mathrm{e}} \mathrm{Mi}$ Jeong Yoon, ${ }^{\mathrm{e}}$ Soo Jeong, ${ }^{\mathrm{a}}$ Young-Uk Cho, ${ }^{\mathrm{d}}$ Sungyang Jo, ${ }^{\mathrm{a}}$ \\ Seung-Bok Lim, ${ }^{\mathrm{e}}$ Joong-Goo Kim, ${ }^{\mathrm{a}}$ Han-Bin Lee, ${ }^{\text {a }}$ Seung Chai Jung, ${ }^{\mathrm{b}}$ Kye Won Park, ${ }^{\mathrm{a}}$ Min-Hwan Lee, ${ }^{\mathrm{a}}$ \\ Dong-Wha Kang, ${ }^{a}$ Dae Chul Suh, ${ }^{\text {b Jong S. Kim }}{ }^{\mathrm{a}}$ \\ aDepartment of Neurology, Asan Medical Center, University of Ulsan College of Medicine, Seoul, Korea \\ 'Department of Emergency Medicine, Asan Medical Center, University of Ulsan College of Medicine, Seoul, Korea \\ 'Department of Radiology, Asan Medical Center, University of Ulsan College of Medicine, Seoul, Korea \\ ${ }^{d}$ Department of Laboratory Medicine, Asan Medical Center, University of Ulsan College of Medicine, Seoul, Korea \\ 'Department of Nursing, Asan Medical Center, Seoul, Korea
}

Background and Purpose Decreasing the time delay for thrombolysis, including intravenous thrombolysis (IVT) with tissue plasminogen activator and intra-arterial thrombectomy (IAT), is critical for decreasing the morbidity and mortality of patients experiencing acute stroke. We aimed to decrease the in-hospital delay for both IVT and IAT through a multidisciplinary approach that is feasible $24 \mathrm{~h} /$ day.

Methods We implemented the Stroke Alert Team (SAT) on May 2, 2016, which introduced hospital-initiated ambulance prenotification and reorganized in-hospital processes. We compared the patient characteristics, time for each step of the evaluation and thrombolysis, thrombolysis rate, and post-thrombolysis intracranial hemorrhage from January 2014 to August 2016.

Results A total of 245 patients received thrombolysis (198 before SAT; 47 after SAT). The median door-to-CT, door-to-MRI, and door-to-laboratory times decreased to $13 \mathrm{~min}, 37.5 \mathrm{~min}$, and $8 \mathrm{~min}$, respectively, after SAT implementation $(P<0.001)$. The median door-to-IVT time decreased from 46 min (interquartile range [IOR] 36-57 $\mathrm{min}$ ) to $20.5 \mathrm{~min}$ (IQR 15.8-32.5 min; $P<0.001$ ). The median door-to-IAT time decreased from $156 \mathrm{~min}$ (IQR 124.5-212.5 $\mathrm{min}$ ) to $86.5 \mathrm{~min}$ (IQR 67.5-102.3 $\min ; P<0.001)$. The thrombolysis rate increased from $9.8 \%(198 / 2,012)$ to $15.8 \%(47 / 297$; $P=0.002)$, and the post-thrombolysis radiological intracranial hemorrhage rate decreased from $12.6 \%(25 / 198)$ to $2.1 \%(1 / 47 ; P=0.035)$.

Conclusions SAT significantly decreased the in-hospital delay for thrombolysis, increased thrombolysis rate, and decreased post-thrombolysis intracranial hemorrhage. Time benefits of SAT were observed for both IVT and IAT and during office hours and after-hours.

Keywords Cerebral infarction; Thrombolytic therapy; Tissue plasminogen activator
Correspondence: Sang-Beom Jeon Department of Neurology, Asan Medical Center, University of Ulsan College of Medicine, 88 Olympic-ro 43-gil, Songpa-gu, Seoul 05505, Korea

Tel: $+82-2-3010-3440$

Fax: +82-2-474-4691

E-mail:sbjeonmd@gmail.com

Received: November 22, 2016

Revised: May 8, 2017

Accepted: May 13, 2017

The authors have no financial conflicts of interest.

\section{Introduction}

Thrombolysis, including intravenous thrombolysis (IVT) with tis- sue plasminogen activator (tPA) and intra-arterial thrombectomy (IAT), is a proven therapy for patients suffering from acute ischemic stroke. ${ }^{1,2}$ However, the benefits of thrombolysis are 
highly time dependent. Earlier thrombolysis is associated with decreased patient mortality and morbidity, and post-thrombolysis intracranial hemorrhage $(\mathrm{ICH}){ }^{3,4}$ Therefore, intensive efforts should be taken to decrease the time to thrombolysis for eligible stroke patients. ${ }^{5}$

The current guidelines state that the door-to-IVT time should be within 60 min of hospital arrival. ${ }^{1}$ A recent national quality improvement initiative in the United States was associated with improved timeliness of tPA administration. ${ }^{6}$ Nevertheless, the goal of a door-to-IVT time within 60 min was achieved in only $40 \%$ of patients treated with IVT. A comprehensive report from Korea also reported a median door-to-IVT time of 56 min. ${ }^{7}$ Surprisingly, the Helsinki model showed a median door-to-IVT time of only 20 min. $^{8}$ However, the success of this model was limited to IVT and was based on a province-wide centralized hospital system. The Melbourne model provided a median door-to-IVT time of $25 \mathrm{~min}$, but out-of-hours delays were $62 \mathrm{~min} .{ }^{9}$ To date, no studies have reported a method to decrease the time delay for both IVT and IAT, and which is feasible $24 \mathrm{~h} /$ day.

We aimed to decrease the in-hospital delay for both IVT and IAT during both after-hours and office hours using a multidisciplinary approach. In addition, we investigated whether the reduction in time to thrombolysis was related to increases in the thrombolysis rate and decrease in the post-thrombolysis $\mathrm{ICH}$ rate.

\section{Methods}

\section{Local emergency medical service system}

Korea has a nationwide, local government-funded emergency medical service (EMS) system. The central EMS dispatch office can be reached on one representative phone number, and then communicates with branch EMS stations that are located closest to callers. However, the EMS does not provide a system by which emergency medical technicians (EMTs) can communicate with the receiving hospitals regarding the care of stroke patients. EMTs usually transport stroke patients to the closest general hospitals. There is no central hospital dedicated to EMS transportation of stroke patients. Therefore, 8 out of 61 EMS stations around Seoul and Gyeonggi-do were in charge of 78\% of EMS transportations of stroke patients to our emergency room (ER) between 2014 and 2015.

\section{Preexisting hospital system}

At our hospital, the evaluation of a stroke, using both computed tomography (CT) and magnetic resonance imaging (MRI) technology, as well as thrombolytic therapies, has been available 24 $\mathrm{h} /$ day for over a decade. MRI was used for routine imaging to screen for cases of acute stroke until 2005. Because of concerns of an increasing door-to-imaging time, we adopted a noncontrast CT scan for imaging screening followed by a multimodal MRI for thrombolysis candidates in 2005. ${ }^{10}$ Intravenous tPA infusions were initiated in the ER and patients were subsequently transported to the MRI room. The MRI room was relocated next to the ER in 2010. The decision whether or not to perform an additional IAT was made after MRI acquisition. Our protocol for $\mathrm{CT}$ and MRI has been detailed previously. ${ }^{11,12} \mathrm{~A}$ computerized physician order entry system for patients experiencing acute stroke was adopted in 2007. ${ }^{13}$ The time window for intravenous tPA was extended to $4.5 \mathrm{~h}$ in January 2014. We performed intra-arterial chemical thrombolysis until 2010; since the introduction of the Penumbra System and stentriever device to our hospital in late 2010, we have been preferentially performing IAT using these devices.

\section{The Stroke Alert Team}

We aimed to decrease the time to thrombolysis. To achieve this goal, in January 2016, we discussed a new model and organized the Stroke Alert Team (SAT), which was comprised of neurologists, emergency physicians, nurses, laboratory physicians and technicians, radiologists, intervention neuroradiologists, radiology technicians and nurses, and admission officers.

Our plan to decrease the thrombolysis time through prospective SAT activities was accepted as the Asan Medical Center Performance Improvement Project 2016. We prepared the SAT project between January 2016 and April 2016 and it was formally initiated on May 2, 2016. As we announced to hospital government and personnel and 8 branches of EMS stations in advance, our specific goals were as follows: (1) to decrease the median door-to-IVT time to $20 \mathrm{~min}$, and (2) to significantly decrease the median door-to-IAT time from that of the past 2 years. We did not specify numbers for the door-to-IAT time because we could not find any references to previous studies or guidelines. We compared the SAT data with the previous data over the past 2 years to define specific goals for motivating SAT members. Moreover, there were no other changes made to the hospital system, structure, or personnel during this period. The SAT program was operational during both office hours and after-hours.

Details of our workflow for the SAT project are described in the Supplemetary Methods and are summarized as follows: (1) we identified the factors causing in-hospital delays; (2) we introduced ambulance prenotification through which EMTs could make direct calls to SAT neurologists $24 \mathrm{~h} /$ day; (3) we reorganized each step, including moving the patients directly to a resuscitation room in the ER, performing point-of-care tests 
(POCT), preparing tPA kits, injecting TPA on the CT table, and the follow-up group messages. We avoided regularly confirming routine laboratory tests, electrocardiography, and chest radiography; (4) advance notification was given to the neuroradiology intervention team. SAT neurologists sent these messages immediately after obtaining the noncontrast $\mathrm{CT}$ scans based on (i) $\mathrm{NIH}$ Stroke Scale scores (NIHSS) of 8 or more; or (ii) observation of cortical symptoms including aphasia, neglect symptoms, and eyeball deviations; and (iii) $<12 \mathrm{~h}$ after symptom onset; and (5) real-time and regular feedback to SAT members were emphasized (Table 1, Supplementary Table 1, Supplementary Figure 1). ${ }^{14}$

\section{Definitions}

The stroke onset was considered to be the time at which the patient developed stroke symptoms or when the patient was last known to be without ischemic symptoms in cases of an uncertain onset time." The IVT time (needle time) was defined as the time when an injection of intravenous IPA was initiated. The IAT time (puncture time) was defined as the time when the intra-arterial catheter was placed in the groin. Post-thromboly- sis ICH was defined as a parenchymal hematoma ( $\mathrm{PH} 1$ and $\mathrm{PH} 2)$ on CT scans performed within $36 \mathrm{~h}$ following thrombolysis. ${ }^{15}$ The CT scans were interpreted by two independent investigators blinded to the clinical data.

\section{Standard protocol approvals, registrations, and patient consent}

This study was approved by the institutional review board of the Asan Medical Center. The need for written informed consent from the patients was waived due to the retrospective nature of this study.

\section{Statistical analysis}

We compared the baseline characteristics of patients, time intervals between each step of the evaluation and management, thrombolysis rates, and post-thrombolysis ICH before and after SAT implementation. We used a Pearson's chi-squared test or Fisher's exact test for categorical variables and a Student's ttest or Mann-Whitney U-test for continuous variables, as appropriate. The interobserver agreement for the interpretation of post-thrombolysis ICH was determined by calculating the kappa

Table 1. The process for acute stroke care in the emergency room before and after implementation of SAT

\begin{tabular}{|c|c|c|}
\hline Measures & Before SAT implementation & After SAT implementation \\
\hline Ambulance prenotification & No & Yes \\
\hline Education and feedback for EMT & No & Yes \\
\hline Hospital registration & Patient or family & Security guard \\
\hline Group message & Yes, once on arrival & Yes, on arrival and follow-up on thrombolysis \\
\hline First place to move patients & Triage at ER & Resuscitation room at ER \\
\hline Blood tests before tPA* & BST, routine labs ${ }^{+}$ & BST, POCT \\
\hline tPA kit & Absent & Prepared \\
\hline Place to inject tPA & Emergency room & $\mathrm{CT}^{*}$ table \\
\hline Person who injects tPA & ER nurse & Neurology resident and fellow \\
\hline tPA permission & Written permission before tPA & $\begin{array}{l}\text { Verbal permission before tPA } \\
\text { Written permission after tPA may be waived }\end{array}$ \\
\hline Advance notification NRI team and SAT & No & Yes, immediately after CT scan, group message \\
\hline Transportation to angiography suite after $\mathrm{MRI}^{\S}$ & Via ER & Directly to angiography suite \\
\hline Prepared IAT set & No & Yes, organized in the angiography suite \\
\hline Thrombolysis protocol & Present, outdated & Present, updated, in detail \\
\hline Time limit in each step & No & Yes \\
\hline Real-time feedback & No & Yes, group message, website \\
\hline Regular case review & No & Yes \\
\hline
\end{tabular}

SAT, the Stroke Alert Team; EMT, emergency medical technician; ER, emergency room; Routine labs, routine laboratory; tPA, tissue plasminogen activator; BST, finger-prick blood sugar test; POCT, point-of-care tests including prothrombin time, hematocrit, and electrolytes; CT, computed tomography; NRI, neuroradiology intervention; MRI, magnetic resonance imaging; IAT, intra-arterial thrombectomy.

${ }^{*}$ Recombinant tPA; ${ }^{\dagger}$ Including complete blood count, prothrombin time, activated partial thromboplastin time, chemical batteries, and electrolyte batteries; ${ }^{\ddagger}$ Noncontrast enhanced $\mathrm{CT}$; ${ }^{5}$ Including diffusion-weighted imaging, fluid-attenuated inversion recovery imaging, gradient-echo imaging, magnetic angiography, and perfusion-weighted imaging. 
coefficient for each investigator. In addition, the baseline characteristics of the thrombolysis patients and time to thrombolysis were grouped by year from March 2005 to August 2016 for demonstrating yearly trends. Statistical significance was defined as a two-tailed $P$-value of $<0.05$. All statistical analyses were performed using SPSS version 21 (IBM Corp., Armonk, NY, USA).

\section{Results}

A total of 1,715 patients experiencing acute ischemic stroke were admitted to our hospital via the ER between January 2014 and December 2015; 297 patients between January 2016 and April 2016; and 297 patients between May 2016 and August 2016. Between January 1, 2014 and August 31, 2016, 247 out of 2,309 patients received thrombolysis for an initial diagnosis of a stroke. Data from one patient with TIA who received IVT before SAT implementation and one patient whose tPA infusion was discontinued because of the identification of an intracranial aneurysm following SAT implementation were included for further analysis. However, data from two patients with stroke mimics (one patient with conversion disorder before SAT implementation, and the other patient with Bell's palsy after SAT implementation) who received IVT were excluded for further analysis. Thus, we performed a statistical analysis for 245 patients who received thrombolysis between two time periods: "before SAT" (from January 1, 2014 to May 1, 2016) vs. "after SAT" (from May 2, 2016 to August 31, 2016). Of the 245 patients that were included in this study, demographic features, initial NIHSS, thrombolysis types, time to each step of evaluation and thrombolysis, and post-thrombolysis ICH are presented in Table 2.

\section{Prehospital activities}

Of the 245 thrombolysis patients, 142 (58.0\%) visited our hospital by EMS. Ambulance prenotification was employed in 13 (59.1\%) out of 22 EMS-transported thrombolysis patients after SAT implementation. In contrast, we received 37 prenotification calls from EMTs after SAT implementation. The final diagnoses of these 37 cases included stroke and TIA for 25 (67.6\%) patients (ischemic stroke: $n=20 ; \mathrm{ICH}: n=4$; and TIA: $n=1$ ). Of 12 nonstroke patients, SAT was activated in seven patients, but not activated in five patients despite prenotification. There were 13 (65\%) out of 20 ischemic stroke patients for whom ambulance prenotification was utilized and that subsequently received thrombolysis.

The median onset-to-door time was $101 \mathrm{~min}$ and this did not change following SAT implementation $(P=0.144)$. The median EMS transportation time was $18 \mathrm{~min}$ and did not change following SAT implementation $(P=0.246)$.

\section{In-hospital activities}

The median door-to-CT time decreased from $18 \mathrm{~min}$ to $13 \mathrm{~min}$ after SAT implementation $(P<0.001)$. The median of door-toMRI time decreased from 56 min to $37.5 \mathrm{~min}$ after SAT implementation $(P<0.001)$. The median time of door-to-prothrombin time (including POCT) decreased from 46 min to 8 min after SAT implementation $(P<0.001)$. However, the time for the reports of routine laboratory tests, including complete blood count $(P=0.426)$ and routine prothrombin time $(P=0.089)$, did not change significantly after SAT implementation.

The median door-to-IVT time decreased from 46 min (interquartile range [IQR] 36-57 $\mathrm{min}$ ) to $20.5 \mathrm{~min}$ (IQR 15.8-32.5 min) following SAT implementation $(P<0.001)$, and the median door-to-IAT time also decreased from $156 \mathrm{~min}$ (IQR 124.5$212.5 \mathrm{~min}$ ) to $86.5 \mathrm{~min}$ (IQR 67.5-102.3 $\mathrm{min} ; P<0.001$ ). As the onset-to-door time remained unchanged, the onset-to-IVT time ( $P=0.024)$ and onset-to-IAT time ( $P=0.002)$ also decreased. Such a reduction in the time for thrombolysis following SAT implementation was remarkable in the context of the recent 11-year trends at our hospital (Supplementary Table 2, Supplementary Figure 2).

The door-to-CT, door-to-MRI, and door-to-laboratory time did not differ between office hours and after-hours ( $P>0.05)$. Similarly, the door-to-IVT time was not different between office hours and after-hours $(P=0.878)$. However, the door-to-IAT time was longer after-hours $(P=0.020)$. Nevertheless, the door-to-IAT time after-hours following SAT implementation (median 100 min; IQR 78-154 min) was shorter than that during office hours before SAT implementation (median 135.5 min; IQR 103.8183.5 min; $P=0.058$; Table 3).

For the 47 patients who visited the ER following SAT implementation, the door-to-IVT time in patients with ambulance prenotification (median $15 \mathrm{~min}$; IQR 12-19 $\mathrm{min}$ ) was shorter than that in patients without ambulance prenotification (median $26 \mathrm{~min} ;$ IQR 20-36 min; $P=0.001$ ) (Supplementary Table 3). However, the door-to-IAT in patients with ambulance prenotification was not significantly shorter than that in patients without ambulance prenotification ( $P=0.473)$. For the 22 patients who visited the ER via EMS ambulance, the door-to-IVT time $(P=0.008)$ and door-to-IAT time $(P=0.044)$ was shorter for the 13 patients whose EMTs utilized prenotification than for the nine patients whose EMTs missed prenotification (Supplementary Figure 3).

\section{Thrombolysis rate}

A total of 198 patients received thrombolysis (IVT only: $n=105$; IAT only: $n=57$; IVT plus IAT: $n=36$ ) before SAT implementation, 
and 47 patients who received thrombolysis (IVT only: $n=28$; IAT only: $n=13$; IVT plus IAT: $n=6$ ) after SAT implementation. The total thrombolysis rate was $9.8 \%(198 / 2,012)$ before SAT implementation and 15.8\% (47/297) after SAT implementation. The IVT rate was 7.0\% $(141 / 2,012)$ before SAT implementation and $11.4 \%(34 / 297)$ after SAT implementation. Therefore, the thrombolysis rate increased significantly following SAT implementation (total thrombolysis rate: $P=0.002$; IVT rate: $P=0.007$ ).

After SAT implementation, advance notification messages were sent to the neuroradiology intervention team in 18 cases, and IAT was performed for 15 patients due to confirmation of major vascular occlusion on magnetic resonance angiography. Advance notification group messages were not sent in 29 cases, but IAT was performed after multimodal MRI scans revealed vessel occlusions in only four of these cases (positive predictive value $=83.3 \%$; negative predictive value $=86.2 \%$ ).

\section{Post-thrombolysis ICH}

Out of 198 patients, 25 (12.6\%) developed ICH before SAT implementation; of these, nine exhibited neurological deteriorations by four or more NIHSS scores (symptomatic ICH: 4.5\%). Out of 47 patients, one (2.1\%) developed ICH after SAT implementation, and none experienced neurological deterioration by four or more NIHSS scores (symptomatic ICH: $0 \%$ ). The ICH rate was decreased after SAT implementation $(P=0.035)$, and the symptomatic ICH rate was not different $(P=0.213)$. The kappa coefficient of the interobserver agreement for $\mathrm{ICH}$ was 0.96 .

Table 2. Baseline characteristics, time to evaluation, time to thrombolysis, and post-thrombolysis intracranial hemorrhage

\begin{tabular}{|c|c|c|c|}
\hline & Before SAT $(n=198)$ & After SAT $(n=47)$ & $P$ \\
\hline Age (years) & $68.5(57.0-76.0)$ & $68.0(64.0-74.0)$ & 0.457 \\
\hline Sex, male & $125(63.1)$ & 28 (59.6) & 0.651 \\
\hline Initial NIHSS & $10.0(6.0-14.0)$ & $9.0(4.0-15.0)$ & 0.838 \\
\hline Ambulance transportation, yes & $120(60.6)$ & $22(46.8)$ & 0.085 \\
\hline Transportation time by EMS (min) & $18.0(14.0-25.0)$ & $17.0(12.5-21.5)$ & 0.246 \\
\hline Stroke onset-to-door (min) & $107.0(45.5-218.5)$ & $91.0(36.0-199.0)$ & 0.144 \\
\hline Door-to-group messaging (min) & $7.0(5.0-11.0)$ & $7.0(3.0-11.75)$ & 0.261 \\
\hline Door-to-CT (min) & $18.0(15.0-23.0)$ & $13.0(8.0-19.0)$ & $<0.001$ \\
\hline Door-to-MRI (min) & $56.0(44.5-70.0)$ & $37.5(30.3-52.0)$ & $<0.001$ \\
\hline Door-to-CBC (min) & $43.0(33.0-59.0)$ & $40.5(28.0-59.3)$ & 0.426 \\
\hline Door-to-PT (routine) (min) & $46.0(37.0-60.0)$ & $41.0(33.8-58.5)$ & 0.089 \\
\hline Door-to-PT (POCT) (min) & $46.0(37.0-60.0)$ & $8.0(4.0-29.0)$ & $<0.001$ \\
\hline \multicolumn{4}{|l|}{ Thrombolysis type } \\
\hline $\mathrm{IVT}^{*}$ only & $105(53.0)$ & $28(59.6)$ & 0.418 \\
\hline IAT only & $57(28.8)$ & $13(27.7)$ & 0.878 \\
\hline $\mathrm{IVT}^{*}$ plus IAT & $36(18.2)$ & $6(12.8)$ & 0.376 \\
\hline Door-to-IVT* (min) & $46.0(36.0-57.0)$ & $20.5(15.8-32.5)$ & $<0.001$ \\
\hline Door-to-IAT (min) & $156.0(124.5-212.5)$ & $86.5(67.5-102.3)$ & $<0.001$ \\
\hline Stroke onset-to-IVT* $(\mathrm{min})$ & 129.0 (84.3-183.3) & 102.5 (49.0-153.5) & 0.024 \\
\hline Stroke onset-to-IAT (min) & $400.0(215.0-805.0)$ & $227.5(153.8-328.5)$ & 0.002 \\
\hline CT-to-IVT* (min) & $27.0(19.0-37.0)$ & $9.0(6.0-15.0)$ & $<0.001$ \\
\hline IVT*-to-IAT (min) & 102.0 (83.5-129.0) & $68.0(65.0-89.0)$ & 0.073 \\
\hline CT-to-MRI (min) & $37.5(23.8-51.3)$ & $25.0(17.0-41.0)$ & 0.003 \\
\hline CT-to-IAT (min) & $134.0(101.0-167.0)$ & $82.0(67.5-119.0)$ & $<0.001$ \\
\hline MRI-to-IAT (min) & $106.0(81.5-167.0)$ & $60.5(49.5-72.3)$ & $<0.001$ \\
\hline Intracranial hemorrhage & $26(13.1)$ & $2(4.3)$ & 0.035 \\
\hline
\end{tabular}

Values are expressed as a number (\%) or median value (interquartile range).

SAT, the Stroke Alert Team; NIHSS, the NIH Stroke Scale; EMS, emergency medical service; CT, computed tomography; MRI, magnetic resonance imaging; CBC, complete blood count; PT, prothrombin time; POCT, point of care test; IVT, intravenous thrombolysis; IAT, intra-arterial thrombectomy.

*With recombinant tissue plasminogen activator. 


\section{Discussion}

We implemented the SAT that was feasible for $24 \mathrm{~h} /$ day as a prospective performance improvement initiative to minimize the in-hospital delay for thrombolysis (i.e., IVT and IAT). Since SAT implementation, the median door-to-IVT time decreased from 46 min to $20.5 \mathrm{~min}$, and the median door-to-IAT time decreased from $156 \mathrm{~min}$ to $86.5 \mathrm{~min}$. We therefore achieved our pre-specified goals to decrease the time for thrombolysis. The total thrombolysis rate increased from 10\% before SAT implementation to $16 \%$ after SAT implementation, and the IVT rate increased from $7 \%$ before SAT implementation to $11 \%$ after
SAT implementation. The post-thrombolysis ICH rate decreased from 13\% before SAT implementation to $2 \%$ after SAT implementation. Thus, SAT activities were potentially beneficial to patients experiencing an acute stroke.

Our prospective project commenced by reviewing the recent data and current status of thrombolysis in our hospital. Based on our recent data, we found that our key measures to be taken were to implement ambulance prenotification and reorganize the in-hospital process. Thus, we introduced ambulance prenotification, updated the thrombolysis protocol, specified time limits for each step, applied POCT, jettisoned unnecessary steps, administered tPA on the CT table, and sent group messages to

Table 3. Baseline characteristics, time to evaluation, time to thrombolysis, and post-thrombolysis intracranial hemorrhage according to the time of hospital arrival following SAT implementation

\begin{tabular}{|c|c|c|c|}
\hline & After-hours $(n=30)$ & Office hours $(n=17)$ & $P$ \\
\hline Age (years) & $68.0(62.0-75.0)$ & $70.0(65.0-74.0)$ & 0.438 \\
\hline Sex, male & $15(50.0)$ & $13(76.5)$ & 0.076 \\
\hline Initial NIHSS & $9.0(4.0-15.0)$ & $10.0(4.5-18.0)$ & 0.478 \\
\hline Ambulance transportation, yes & $15(50.0)$ & $7(41.2)$ & 0.560 \\
\hline Ambulance prenotification, yes & $8(26.7)$ & $5(29.4)$ & 1.000 \\
\hline Transportation time by EMS (min) & $16.0(13.0-21.0)$ & $20.0(14.0-25.5)$ & 0.395 \\
\hline Prenotification-to-door (min) & $11.5(9.3-14.5)$ & $11.0(7.5-17.0)$ & 1.000 \\
\hline Stroke onset-to-door (min) & $90.0(34.8-159.3)$ & $100.0(34.0-214.0)$ & 0.790 \\
\hline Door-to-group messaging (min) & $8.0(3.0-13.5)$ & $4.5(2.0-8.5)$ & 0.173 \\
\hline Door-to-CT (min) & $14.0(8.0-19.3)$ & $10.0(7.8-17.5)$ & 0.585 \\
\hline Door-to-MRI (min) & $39.0(33.0-53.0)$ & $31.0(23.0-51.0)$ & 0.131 \\
\hline Door-to-CBC (min) & $49.0(30.5-59.5)$ & $37.0(25.5-57.0)$ & 0.406 \\
\hline Door-to-PT (routine) (min) & $40.0(32.0-55.0)$ & $43.0(39.0-60.5)$ & 0.127 \\
\hline Door-to-PT (POCT) (min) & $8.0(4.0-26.5)$ & $9.0(3.0-42.0)$ & 0.665 \\
\hline \multicolumn{4}{|l|}{ Thrombolysis type } \\
\hline IVT* only & $20(66.7)$ & $8(47.1)$ & 0.188 \\
\hline IAT only & 5 (16.7) & $8(47.1)$ & 0.041 \\
\hline IVT* plus IAT & $5(16.7)$ & $1(5.9)$ & 0.396 \\
\hline Door-to-IVT* (min) & $22.0(15.5-31.5)$ & $19.0(14.5-46.0)$ & 0.878 \\
\hline Door-to-IAT (min) & $100.0(78.0-154.0)$ & $72.0(51.0-89.0)$ & 0.020 \\
\hline Stroke onset-to-IVT* $(\mathrm{min})$ & $118.0(74.5-158.0)$ & $57.0(42.5-111.5)$ & 0.066 \\
\hline Stroke onset-to-IAT (min) & $195.0(147.0-315.0)$ & $246.0(148.5-544.5)$ & 0.603 \\
\hline CT-to-IVT* (min) & $8.0(6.0-15.0)$ & $9.0(6.3-12.0)$ & 0.842 \\
\hline IVT*-to-IAT (min) & 73.0 (63.5-101.3) & $67.0(67.0-67.0)$ & 0.857 \\
\hline CT-to-MRI (min) & $35.0(20.3-50.0)$ & $32.0(23.0-51.0)$ & 0.742 \\
\hline CT-to-IAT (min) & $135.5(104.8-167.8)$ & $99.0(74.0-143.0)$ & 0.002 \\
\hline MRI-to-IAT (min) & $66.0(46.5-83.5)$ & $56.5(47.0-67.8)$ & 0.562 \\
\hline Intracranial hemorrhage & $2(6.7)$ & $0(0)$ & 0.528 \\
\hline
\end{tabular}

Values are expressed as a number (\%) or median value (interquartile range).

SAT, the Stroke Alert Team; NIHSS, the NIH Stroke Scale; EMS, emergency medical service; CT, computed tomography; MRI, magnetic resonance imaging; CBC, complete blood count; PT, prothrombin time; POCT, point of care test; IVT, intravenous thrombolysis; IAT, intra-arterial thrombectomy.

*With recombinant tissue plasminogen activator. 
SAT members for each step. These comprehensive activities and real-time peer reviews were feasible, did not require any changes to the overall hospital structure and manpower, and were effective for each step of the evaluation and management processes.

Our success in decreasing the time delay was likely not accomplished in only 4 months, and long-standing continued efforts to improve the hospital system underlie the current achievements. We previously demonstrated a significant reduction in the door-to-IVT time by changing our MRI screening system to CT-plus-MRI screening in December 2005, and by introducing a computerized physician order entry system for thrombolysis candidates in 2007..$^{10,13}$ In addition, we relocated a new MRI machine beside the ER in 2010. Stroke center protocols were also updated every few years. Nevertheless, our current data showing a reduction in the door-to-thrombolysis time is remarkable in comparison with our previous efforts over the past decade.

The SAT was designed to be active $24 \mathrm{~h} /$ day, and no differences regarding the door-to-CT/MRI time, door-to-laboratory time, and door-to-IVT time were found between the office hours and after-hours after SAT implementation. However, the door-to-IAT time was longer after-hours than during office hours. Nevertheless, the door-to-IAT time after-hours following SAT implementation was shorter than that during the office hours prior to SAT implementation. Since other variables than the door-to-IAT time and CT-to-IAT time were delayed afterhours, we believe that the majority of these delays were due to the time required for the neuroradiologists to arrive at the hospital at night and on holidays. These findings suggest that interventions including the neuroradiologist's early involvement in the assessment of thrombolysis candidates are also imperative as typically they do not remain at the hospital after-hours. For this purpose, advance notification provided to the intervention neuroradiologists may be helpful. Neurologist criteria for advance notification exhibited high positive (83\%) and negative predictive values $(86 \%)$ in the present study. However, further studies are required to identify proper methods to further decrease the door-to-IAT time, especially after-hours. Earlier initiation of IAT is associated with decreased mortality and morbidity. ${ }^{2,16}$ However, no previous studies have reported efforts to decrease the door-to-IAT time. The median door-to-IAT time of $86.5 \mathrm{~min}$ in this study is real-world data and shorter than the times in any other recently published clinical trials. ${ }^{16-19}$ We performed IAT in consecutive patients after-hours as well as during office hours.

Our study has some limitations that should be noted. First, the main outcomes of this study are the reduction in time to thrombolysis for the most recent 4-month period compared with the previous 28 months. A period of 4 months was sufficient to demonstrate improved temporal profiles, but not long enough to investigate clinical outcomes, such as the 3-month modified Rankin Scale. However, the therapeutic benefit of thrombolysis is the greatest when administered early after a stroke and declines over time. Lower rates of ICH after SAT implementation in this study may reduce patient morbidity and mortality. Our future studies will therefore investigate whether the SAT system can be maintained beyond several months and whether the saved time is related to better clinical outcomes. Second, because the hospital-initiated ambulance prenotification was not supported by the government or central EMS, we were required to contact each satellite EMS station and were only able to introduce prenotification in eight out of the 61 EMS stations. As a result, ambulance prenotification could only be utilized in $<60 \%$ of the total EMS transportations. Simply using EMS to arrive at the hospital is not sufficient for thrombolysis; however, ambulance prenotification does enhance timely thrombolysis. ${ }^{20-22}$ We showed that prenotification calls reduced the door-to-IVT time through reduction in door-to-CT time and door-to-POCT time. Therefore, government or a central EMS-initiated prenotification system is highly warranted. Third, imaging protocols could affect the time to thrombolysis, especially to IAT. We previously demonstrated that changing the imaging protocol from MRI to CT screening decreased the doorto-IVT time. ${ }^{10}$ Similarly, the change in the imaging protocol from multimodal MRI screening into $\mathrm{CT}$ with $\mathrm{CT}$ angiography screening may additionally decrease the door-to-IAT time; further studies are required to address this issue. Fourth, although our SAT activities resulted in a significant reduction in the time for thrombolysis, this may not be directly applicable to other hospitals. In the Helsinki and Melbourne models, for instance, patients went straight to a CT room on ambulance stretchers, whereas we moved patients to the resuscitation room upon arrival and finished basic evaluations within 5 min. Five minutes was enough time to clear the CT room during the patients' stay in the resuscitation room. Moving unstable patients directly to a CT table can be dangerous. We therefore believe that reorganization of the in-hospital process for thrombolysis should be done according to the specific situation of each hospital. Fifth, we performed POCT in most patients before infusion of $\mathrm{PAA}$, which was followed by routine laboratory tests. However, POCT tests in the setting of thrombolytic therapies need to be validated in future studies. 


\section{Conclusions}

Using a multidisciplinary approach, we showed that SAT significantly reduced in-hospital delay for thrombolysis treatment. SAT activity was also related to increased thrombolysis rate and decreased post-thrombolysis ICH. Time benefits of SAT were observed for both IVT and IAT, during both office hours and afterhours. Further studies to investigate whether reduction of inhospital delay is related to better clinical outcomes are needed.

\section{Acknowledgments}

We are grateful to EMTs, emergency physicians and nurses, neurology residents, radiology fellows, radiology technicians and nurses, laboratory technicians, security guards, office administrators, and neurological intensive care unit nurses.

\section{Supplementary Materials}

Supplementary materials related to this article can be found online at https://doi.org/10.5853/jos.2016.01802.

\section{References}

1. Jauch EC, Saver JL, Adams HP Jr, Bruno A, Connors JJ, Demaerschalk BM, et al. Guidelines for the early management of patients with acute ischemic stroke: a guideline for healthcare professionals from the American Heart Association/American Stroke Association. Stroke 2013;44:870-947.

2. Hong KS, Ko SB, Lee JS, Yu KH, Rha JH. Endovascular recanalization therapy in acute ischemic stroke: updated metaanalysis of randomized controlled trials. J Stroke 2015;17: 268-281.

3. Saver JL, Fonarow GC, Smith EE, Reeves MJ, Grau-Sepulveda MV, Pan $W$, et al. Time to treatment with intravenous tissue plasminogen activator and outcome from acute ischemic stroke. JAMA 2013;309:2480-2488.

4. Lees KR, Bluhmki E, von Kummer R, Brott TG, Toni D, Grotta $J C$, et al. Time to treatment with intravenous alteplase and outcome in stroke: an updated pooled analysis of ECASS, ATLANTIS, NINDS, and EPITHET trials. Lancet 2010;375:16951703.

5. Fassbender K, Balucani C, Walter S, Levine SR, Haass A, Grotta J. Streamlining of prehospital stroke management: the golden hour. Lancet Neurol 2013;12:585-596.

6. Fonarow GC, Zhao X, Smith EE, Saver JL, Reeves MJ, Bhatt $\mathrm{DL}$, et al. Door-to-needle times for tissue plasminogen activator administration and clinical outcomes in acute ischemic stroke before and after a quality improvement initiative. JAMA 2014;311:1632-1640.

7. Hong KS, Bang OY, Kim JS, Heo JH, Yu KH, Bae HJ, et al. Stroke statistics in Korea: part II stroke awareness and acute stroke care, a report from the Korean Stroke Society and Clinical Research Center for Stroke. J Stroke 2013;15:67-77.

8. Meretoja A, Strbian D, Mustanoja S, Tatlisumak T, Lindsberg PJ, Kaste M. Reducing in-hospital delay to 20 minutes in stroke thrombolysis. Neurology 2012;79:306-313.

9. Meretoja A, Weir L, Ugalde M, Yassi N, Yan B, Hand P, et al. Helsinki model cut stroke thrombolysis delays to 25 minutes in Melbourne in only 4 months. Neurology 2013;81:10711076.

10. Yoo SH, Kwon SU, Lee DH, Kim SJ, Kim JS, Kang DW. Comparison between MRI screening and CT-plus-MRI screening for thrombolysis within $3 \mathrm{~h}$ of ischemic stroke. J Neurol Sci 2010;294:119-123.

11. Jeon SB, Kwon SU, Cho AH, Yun SC, Kim JS, Kang DW. Rapid appearance of new cerebral microbleeds after acute ischemic stroke. Neurology 2009;73:1638-1644.

12. Jeon SB, Kwon SU, Park JC, Lee DH, Yun SC, Kim YJ, et al. reduction of midline shift following decompressive hemicraniectomy for malignant middle cerebral artery infarction. $J$ Stroke 2016;18:328-336.

13. Heo JH, Kim YD, Nam HS, Hong KS, Ahn SH, Cho HJ, et al. A computerized in-hospital alert system for thrombolysis in acute stroke. Stroke 2010;41:1978-1983.

14. Harbison J, Hossain O, Jenkinson D, Davis J, Louw SJ, Ford GA. Diagnostic accuracy of stroke referrals from primary care, emergency room physicians, and ambulance staff using the face arm speech test. Stroke 2003;34:71-76.

15. Fiorelli $M$, Bastianello $S$, von Kummer $R$, del Zoppo GJ, Larrue $V$, Lesaffre $E_{\text {, et al. Hemorrhagic transformation within } 36}$ hours of a cerebral infarct: Relationships with early clinical deterioration and 3-month outcome in the European Cooperative Acute Stroke Study I (ECASS I) cohort. Stroke 1999; 30:2280-2284.

16. Fransen $P S$, Berkhemer $O A$, Lingsma $H F$, Beumer $D$, van den Berg $L A, Y o o A J$, et al. Time to reperfusion and treatment effect for acute ischemic stroke: a randomized clinical trial. JAMA Neurol 2016;73:190-196.

17. Saver JL, Goyal M, Bonafe A, Diener HC, Levy El, Pereira VM, et al. Stent-retriever thrombectomy after intravenous t-PA vs. t-PA alone in stroke. N Engl J Med 2015;372:2285-2295.

18. Mocco J, Zaidat 00, von Kummer R, Yoo AJ, Gupta R, Lopes $D$, et al. Aspiration thrombectomy after intravenous alteplase versus intravenous alteplase alone. Stroke 2016;47:23312338. 
19. Jovin TG, Chamorro A, Cobo $E$, de Miquel MA, Molina $C A$, Rovira $A$, et al. Thrombectomy within 8 hours after symptom onset in ischemic stroke. N Engl J Med 2015;372:2296-2306.

20. Kim SK, Lee SY, Bae HJ, Lee YS, Kim SY, Kang MJ, et al. Pre-hospital notification reduced the door-to-needle time for iv t-PA in acute ischaemic stroke. Eur J Neurol 2009;16:1331-1335.

21. Bae HJ, Kim DH, Yoo NT, Choi JH, Huh JT, Cha JK, et al. Prehospital notification from the emergency medical service re- duces the transfer and intra-hospital processing times for acute stroke patients. J Clin Neurol 2010;6:138-142.

22. Jeong JH, Yang JY, Cha JK, Kim DH, Nah HW, Kang MJ, et al. Feasibility of a pre-hospital notification system using direct calls from paramedics of 119 EMS ambulances for acute stroke patients: accuracy of diagnosis and efficacy of shortening of door-to-imaging time. J Neurocrit Care 2015;8:98102. 
Supplementary Methods 1. Workflow for the Stroke Alert Team project.

First, we identified factors causing an in-hospital delay for thrombolysis. These included a lack of preparation for ambulance arrival; delayed group messaging upon patient arrivals by emergency physicians; delayed examinations by neurologists; mandatory confirmation of laboratory test results prior to tissue plasminogen activator (tPA) infusion; obtaining written consent for tPA; lack of prepared tPA sets; outdated thrombolysis protocol; occasional long discussion on difficult cases; delayed acquisition of MRI; delayed discussion with intervention neuroradiologists; inefficient transportation flow among emergency room (ER), computed tomography (CT) room, magnetic resonance imaging (MRI) room, and angiography suite; and lack of prepared intra-arterial thrombectomy (IAT) sets in the angiography suite.

Second, we realized that we required ambulance prenotification to save time for thrombolysis preparation. Thus, we introduced a phone call by which emergency medicine technicians (EMTs) could make direct calls to the Stroke Alert Team (SAT) neurologists $24 \mathrm{~h} /$ day. We requested that EMTs call us whenever they were transporting stroke patients to our hospital. For this purpose, we provided every EMT from eight EMS stations repeated education on stroke symptoms, the face arm speech time (FAST) scale, representative phone numbers ("hot line"), and our SAT system (Supplemetary Table 1). We created posters describing the FAST scale and distributed them to EMTs who attended the education sessions and EMS stations, which were to be posted on each ambulance and EMS station (Supplementary Figure 1). We also attempted to obtain histories from the patients' families via telephone before ambulance arrival whenever possible.

Third, we reorganized each step as shown in Table 1. Upon ambulance arrival, the patients bypassed triage and moved directly to a resuscitation room, a dedicated space for the management of critically ill patients (e.g., cardiac arrest) in the ER, while the security guards made a hospital registration on behalf of the patients' families. In the resuscitation room, several emergency nurses checked vital signs, obtained finger-stick blood samples, and inserted a venous access to obtain blood samples and infused normal saline, while neurologists examined the patients, and emergency physicians activated the computerized physician order entry system and emptied the CT room. We introduced a device for point-of-care tests (POCT) for prothrombin time (the CoaguChek ${ }^{\circledR}$ XS Pro, Roche, Switzerland) and began to utilize a preexisting POCT device for glucose, electroIyte battery, a chemical battery, and hematocrits in the resuscitation room. Methods of delivering and testing routine blood samples were not altered, but conspicuous labels and audible alarms for blood samples were supplied to the laboratory technicians. The time limit for the patients to stay in the resuscitation room was $5 \mathrm{~min}$, and the patients were to be transported to the CT room, which was emptied following the patients' arrival. The tPA kit contained the tPA vial, catheter, alcohol swab, gauze, and manual for tPA injection, and was prepared before transportation to the CT room. Verbal informed consent for IPA was obtained from patients and their families, and written informed consent was deferred when the patients' families were not present and the patients were not able to communicate following the initiation of a bolus injection of tPA. Neurologists mixed and injected TPA on the CT table immediately after obtaining the $\mathrm{CT}$ images. Follow-up group messages were sent to SAT members after the injection of tPA. Confirmation of routine laboratory tests, electrocardiography, and chest radiography were deferred after initiation of a maintenance infusion of tPA unless the patients experienced chest pain, hypotension, pallor, an uncertain medical history, and a history of malignancy, hematological disease, severe liver disease, or other contraindications to thrombolysis. The injection of tPA was also deferred after the MRI scan in cases of diagnostic uncertainty and an unclear risk-benefit ratio of thrombolysis. We requested that the MRI room be emptied within 5 min.

Fourth, we updated the written protocol for thrombolysis, which specified that NIH Stroke Scale (NIHSS) scores of 2 to 25 were to be included and an age of 81 years or more was to be excluded for intravenous thrombolysis (IVT) in accordance with the Korean Ministry of Food and Drug Safety approval and the Korean Health Insurance Review and Assessment Service guidelines. We also predefined strategies for dealing with difficult cases, such as pregnant woman, intracranial aneurysms, thoracoabdominal aneurysms, tumors, and endocarditis. If the patients did not arrive at the ER via ambulance or the EMTs missed prenotification, we could not prepare for patient arrivals, but other SAT processes were the same as those previously mentioned. No additional equipment, structures, or personnel were employed, except a cellular phone for the prenotification call and a POCT device for prothrombin time since SAT implementation.

Fifth, strategies to obtain an earlier initiation of IAT were also employed and included the neurologists' advance notification group messages to the neuroradiology intervention team and other SAT members, written protocol for IAT, and prepared IAT sets. SAT neurologists sent advance notification group messages for probable IAT candidates immediately after obtaining the noncontrast CT scans based on following criteria: (1) NIHSS were 8 or higher; or (2) cortical symptoms including aphasia, neglect symptoms, and eyeball deviations were observed; and 
(3) it was $<12 \mathrm{~h}$ after symptom onset. A written protocol for IAT included computer passwords in an angiography suite, as well as technical tips for duty attending intervention neuroradiologists, radiology fellows, and technicians. Prepared IAT sets were placed on a dedicated shelf in the angiography suite.

Sixth, feedback to SAT members was also applied. The SAT team leader (S.-B. J) provided real-time feedback to the SAT members. In addition, he reviewed every thrombolysis candidate and ambulance prenotification case with the SAT members during weekly meetings. SAT data were also posted in the online bulletin and were updated daily for SAT members. 
Supplementary Table 1. Factors to enhance ambulance prenotification by EMTs

\begin{tabular}{|c|c|}
\hline Simplified call system & $\begin{array}{l}\text { We adopted a hotline with a single phone number. } \\
\text { This hotline called our team and did not involve smart phone applications or webcam chatting. }\end{array}$ \\
\hline Efforts not to miss calls from EMTs & $\begin{array}{l}\text { In any case of missed calls by our responder, the call was automatically forwarded to another } \\
\text { responder. }\end{array}$ \\
\hline Reassuring EMTs on our care system & $\begin{array}{l}\text { We promised EMTs that we would do our best to treat stroke victims. } \\
\text { We waited for EMTs when they were transferring patients to our emergency room. } \\
\text { We reassured EMTs that we always save beds dedicated to patients receiving thrombolytic } \\
\text { therapies in our neurological intensive care unit. }\end{array}$ \\
\hline Repeated education of EMTs & $\begin{array}{l}\text { We explained details of our SAT activities to EMTs. } \\
\text { We presented SAT data to EMTs during the education. } \\
\text { We used a poster and video clips on FAST during the education (Supplementary Figure 1). }\end{array}$ \\
\hline Formal letters to EMS stations & We sent EMS stations formal letters on education sessions provided by the SAT leader. \\
\hline Positive feedback from patients and families & $\begin{array}{l}\text { During the education, we showed EMTs video clips and letters, in which patients and their } \\
\text { families appreciated the ambulance prenotification call. }\end{array}$ \\
\hline Positive feedback from our stroke team & We issued certificates for EMTs who used ambulance prenotification calls. \\
\hline
\end{tabular}

EMT, emergency medicine technician; EMS, emergency medical service. 


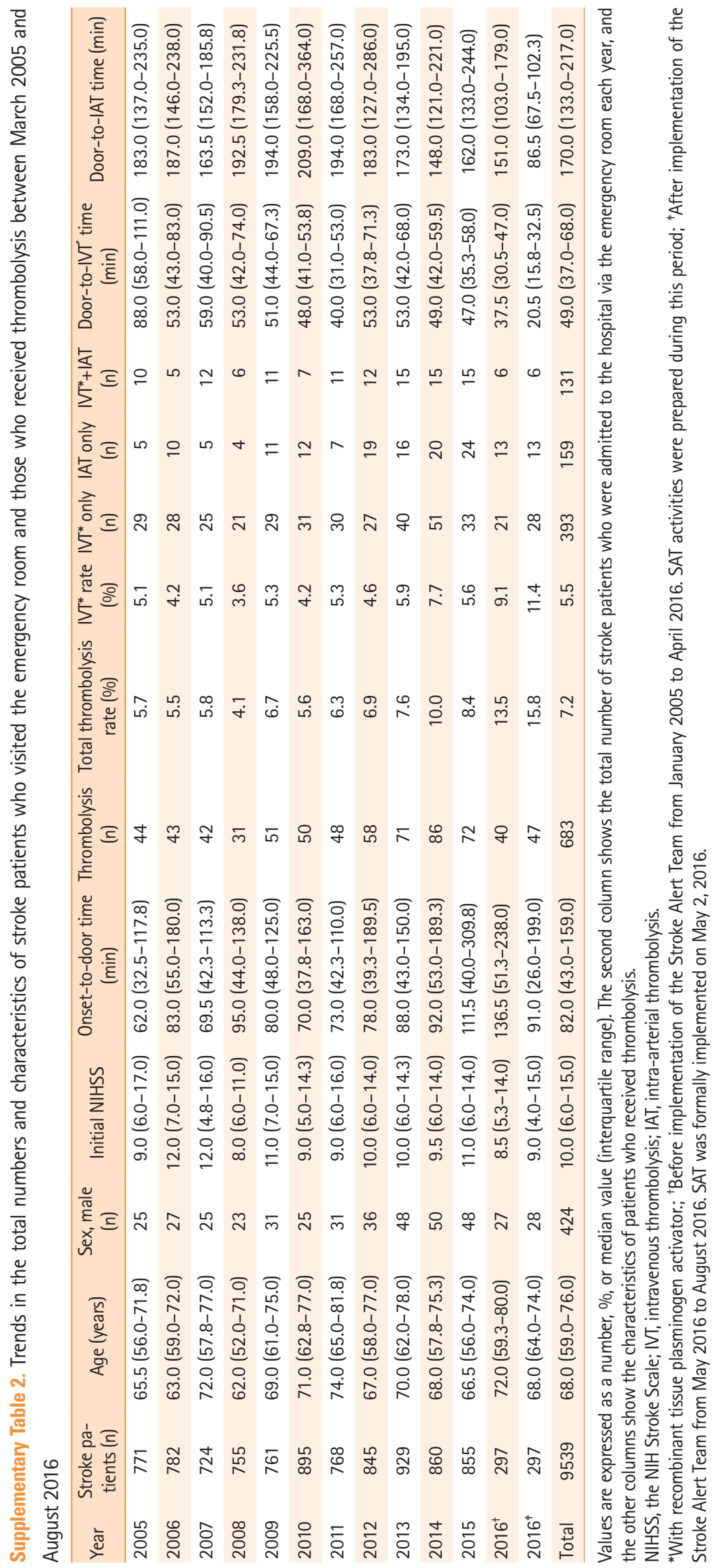


Supplementary Table 3. Baseline characteristics, time to evaluation, and time to thrombolysis according to the ambulance prenotification

\begin{tabular}{|c|c|c|c|}
\hline & $\begin{array}{c}\text { Prenotification }(-) \\
(n=34)\end{array}$ & $\begin{array}{c}\text { Prenotification }(+) \\
(n=13)\end{array}$ & $P$ \\
\hline Age (years) & $70.0(62.8-74.3)$ & $68.0(64.5-74.0)$ & 0.802 \\
\hline Sex, male & & & 0.324 \\
\hline Initial NIHSS & $8.0(3.8-16.3)$ & $10.0(8.5-13.5)$ & 0.535 \\
\hline Stroke onset-to-door ( $\mathrm{min}$ ) & $97.0(45.3-152.8)$ & $89.0(29.0-218.0)$ & 0.887 \\
\hline Door-to-group messaging ( $\mathrm{min}$ ) & $7.0(4.0-12.0)$ & $3.0(2.0-11.5)$ & 0.028 \\
\hline Door-to-CT (min) & $17.0(12.0-20.0)$ & $7.0(5.0-9.0)$ & $<0.001$ \\
\hline Door-to-MRI (min) & $38.0(30.0-62.0)$ & $34.0(30.5-46.5)$ & 0.403 \\
\hline Door-to-CBC (min) & $49.0(30.5-61.5)$ & $32.0(22.0-43.0)$ & 0.016 \\
\hline Door-to-PT (routine) (min) & $46.0(39.0-63.5)$ & $34.0(30.5-47.5)$ & 0.018 \\
\hline Door-to-PT (POCT) (min) & $10.0(7.0-20.5)$ & $2.0(1.0-4.0)$ & $<0.001$ \\
\hline \multicolumn{4}{|l|}{ Thrombolysis type } \\
\hline IVT only & $22(64.7)$ & $6(46.2)$ & 0.324 \\
\hline IAT only & $11(32.4)$ & $2(15.4)$ & 0.301 \\
\hline IVT plus IAT & $1(2.9)$ & $5(38.5)$ & 0.004 \\
\hline Door-to-IVT (min) & $26.0(20.0-36.0)$ & $15.0(12.0-19.0)$ & $<0.001$ \\
\hline Door-to-IAT (min) & $179.0(46.0-137.5)$ & 89.5 (78.3-99.3) & 0.473 \\
\hline Stroke onset-to-IVT (min) & $113.0(80.0-152.0)$ & $57.0(45.0-158.0)$ & 0.363 \\
\hline Stroke onset-to-IAT (min) & $227.5(174.8-281.5)$ & $252.0(112.0-655.3)$ & 1.000 \\
\hline CT-to-IVT (min) & $10.5(6.0-16.5)$ & $6.0(6.0-9.0)$ & 0.157 \\
\hline IVT-to-IAT (min) & $78.0(78.0-78.0)$ & 67.5 (63.5-101.3) & 0.857 \\
\hline CT-to-MRI (min) & $21.0(12.5-39.8)$ & $28.0(24.5-41.0)$ & 0.198 \\
\hline CT-to-IAT (min) & 76.5 (35.0-143.5) & $82.0(71.0-93.8)$ & 0.798 \\
\hline MRI-to-IAT (min) & $60.0(49.5-74.8)$ & $61.5(38.0-72.3)$ & 0.959 \\
\hline
\end{tabular}

NIHSS, the NIH Stroke Scale; CT, computed tomography; MRI, magnetic resonance imaging; CBC, complete blood count; PT, prothrombin time; POCT, point of care test; IVT, intravenous thrombolysis; IAT, intra-arterial thrombectomy. 


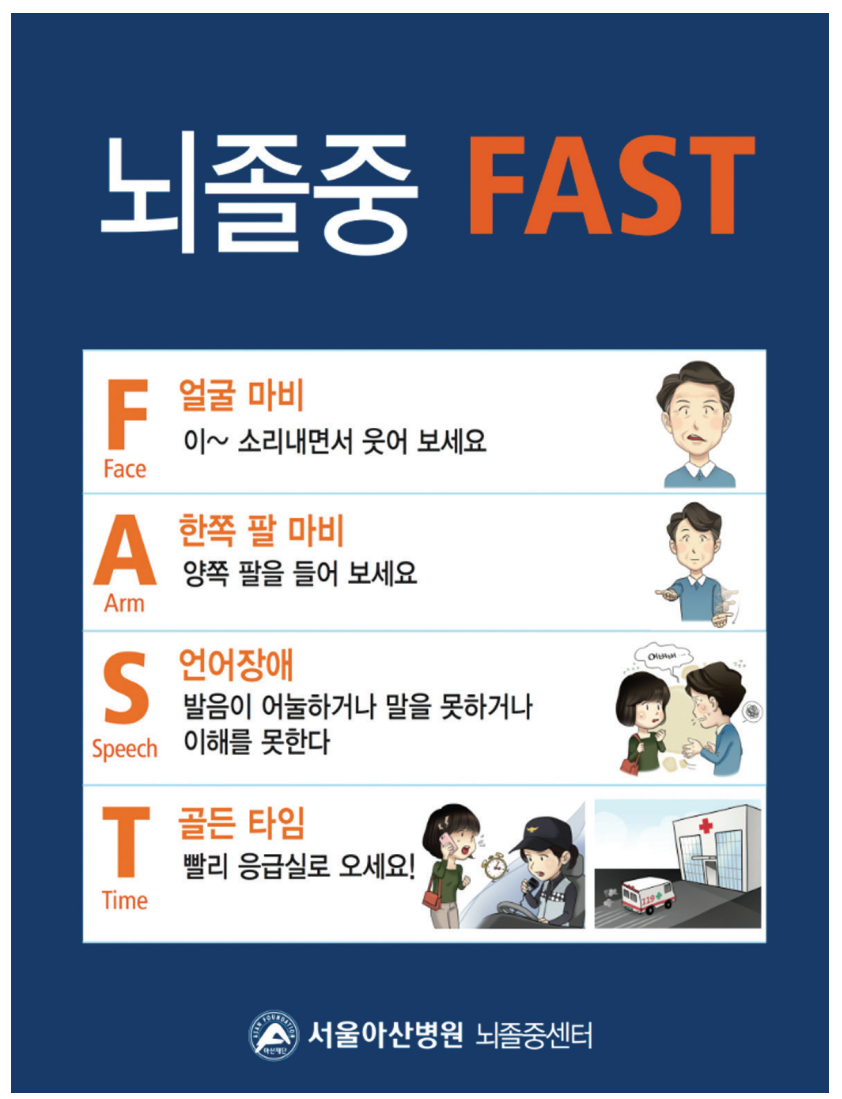

Supplementary Figure 1. The poster on the face arm speech time scale to educate emergency medical technicians. We created posters on the face arm speech time scale (FAST) scale to distribute to emergency medical technicians and local emergency medical service stations. These posters were posted on each ambulance and emergency medical service station. The Stroke Alert Team (SAT) phone numbers, hospital name, and hospital logo were not shown in the original version of this poster for local legal reasons. 

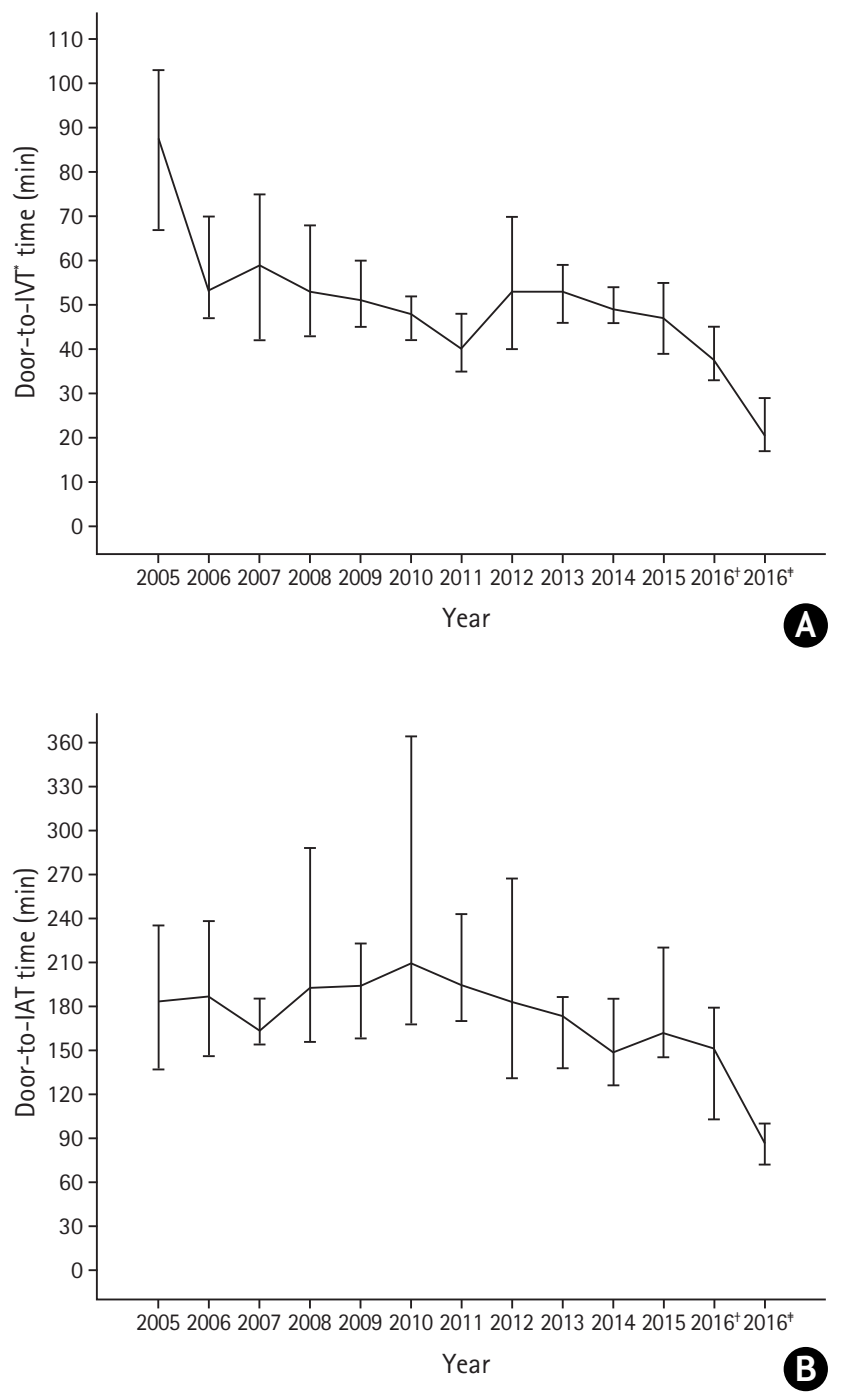

Supplementary Figure 2. In-hospital delay in administering thrombolysis. The 11-year trends of the median door-to-IVT time (A) and door-to-IAT time (B). Since SAT implementation (May 2, 2016), the median door-to-IVT time decreased to $20.5 \mathrm{~min}$, and the median door-to-IAT time was also decreased to $86.5 \mathrm{~min}$. SAT activities were prepared between January 1, 2016 and May 1, 2016, and implemented between May 2, 2016 and August 31, 2016. Error bars represent medians and 95\% confidence intervals. IVT, intravenous thrombolysis; IAT, intra-arterial thrombectomy; SAT, the Stroke Alert Team. *With recombinant tissue plasminogen activator; ${ }^{+}$Between January 1, 2016 and May 1, 2016 (run-up period); ${ }^{*}$ Between May 2, 2016 and August 31, 2016 (after SAT implementation). 


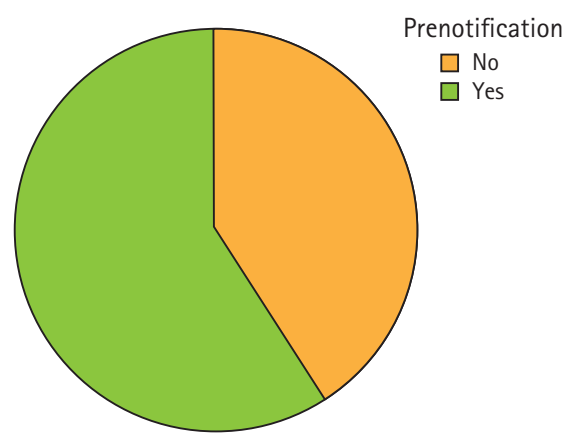

A

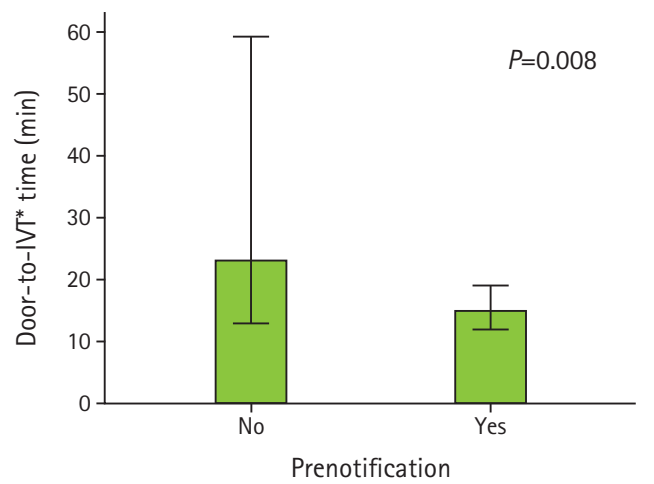

B

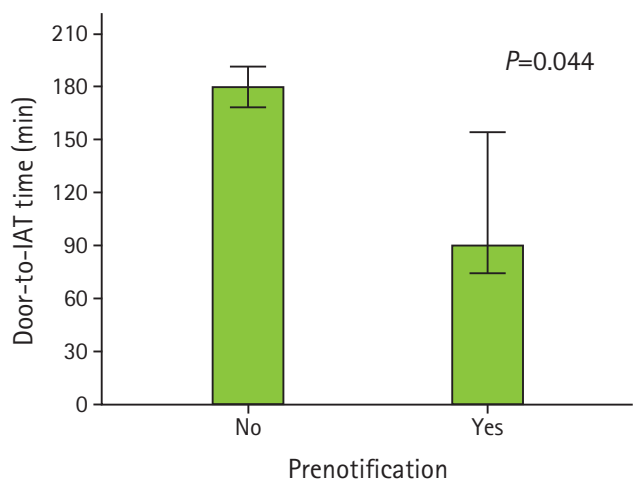

C

Supplementary Figure 3. Ambulance prenotification utilization in 22 thrombolysis patients who arrived at the hospital by EMS (A). The median door-to-IVT time and door-to-IAT time in 22 patients whose emergency medical technicians utilized ambulance prenotification (B and C). EMS, emergency medical service; IVT, intravenous thrombolysis; IAT, intra-arterial thrombectomy. *With recombinant tissue plasminogen activator. 